The majority were in clusters with $<5$ cases and therefore did not reach the HPA cluster investigation threshold of $=5$ cases in 24 months. Data will be presented for the entirety of 2010, therefore numbers are subject to change.

\section{P51 TUBERCULOSIS OUTCOME FOLLOWING PRE-TREATMENT ASSESSMENT FOR DIRECTLY OBSERVED OR SELF- ADMINISTERED THERAPY: STILL ROOM FOR IMPROVEMENT?}

doi:10.1136/thoraxjnl-2011-201054c.51 well within NCL TB service, but were too often lost to follow-up if transferred elsewhere. It is unclear whether this reflects inadequate local data collection and communication by, and with, our service, or genuine loss from healthcare. Either way, this requires urgent attention. The planned introduction of enhanced case management within the London TB model of care may improve this.

\section{P52 RISING PAEDIATRIC TUBERCULOSIS IN GREATER MANCHESTER: EPIDEMIOLOGY AND BCG VACCINATION STATUS OF CASES}

doi:10.1136/thoraxjnl-2011-201054c.52

J C Barrett, S Dart, A Solamalai, C Snook, M Lipman. Royal Free Hospital, London, UK

Setting and Methods London has high rates and large numbers of TB notifications. Treatment completion is generally $<85 \%$ (the figure recommended by the WHO to achieve effective control). NICE TB guidelines (2011) advise using risk assessment to identify those individuals most likely to non-adhere to therapy and hence who require enhanced case management, including directly observed therapy (DOT), to complete treatment successfully. The North Central London TB Network piloted a risk assessment tool, derived from reported risk factors predisposing to non-adherence plus information from a patient profile study undertaken across London, in a cohort of $306 \mathrm{~TB}$ patients starting treatment between June and December 2008. On the basis of the individual's risk of non-adherence score they were broadly allocated to DOT or self-administered therapy (SAT). Here we evaluate treatment outcomes (completion and need for re-treatment) using the London TB register (LTBR) and individual case records.

Results Subjects receiving SAT had excellent treatment completion rates (91\%), with 3\% lost to TB service follow-up (Abstract P51 table 1). Those on DOT had a lower completion rate-which at $80 \%$ was less than the international standard. Ten per cent of DOT subjects were lost to follow-up (all after transfer out of NCL TB service care). Death rates were threefold higher in the DOT group. After 20 months median follow-up post treatment completion, 3 SAT and 0 DOT patients had been re-treated for TB.

\section{Abstract P51 Table 1}

\begin{tabular}{|c|c|c|c|c|}
\hline \multirow[b]{2}{*}{ Completed in NCL TB service } & \multicolumn{2}{|c|}{ DOT $(n=30)$} & \multicolumn{2}{|c|}{ SAT $(n=276)$} \\
\hline & 21 & $70.0 \%$ & 233 & $84.4 \%$ \\
\hline \multicolumn{5}{|l|}{ Died } \\
\hline TB & 1 & & 2 & \\
\hline Not TB associated & 0 & & 4 & \\
\hline Unknown & 1 & $6.7 \%$ & 0 & $2.2 \%$ \\
\hline Lost to follow-up & 0 & & 5 & $1.8 \%$ \\
\hline Stopped & 1 & $3.3 \%$ & 8 & $2.9 \%$ \\
\hline \multicolumn{5}{|l|}{ Transferred out } \\
\hline \multicolumn{5}{|l|}{ LTBR } \\
\hline Completed & 0 & & 12 & $4.3 \%$ \\
\hline Lost to follow-up & 1 & $3.3 \%$ & 0 & \\
\hline \multicolumn{5}{|l|}{ Non LTBR } \\
\hline Completed & 3 & $10.0 \%$ & 4 & $1.4 \%$ \\
\hline Lost to follow-up & 2 & $6.7 \%$ & 1 & $0.3 \%$ \\
\hline \multicolumn{5}{|l|}{ Overseas } \\
\hline Completed & 0 & & 1 & $0.3 \%$ \\
\hline Lost to follow-up & 0 & & 6 & $2.2 \%$ \\
\hline Total completed & 24 & $80.0 \%$ & 250 & $90.5 \%$ \\
\hline
\end{tabular}

Conclusions The risk assessment tool appears to discriminate those patients who can receive SAT; though it should be noted that retreatment was only required in this group-suggesting possible poor adherence with therapy in some individuals. Subjects on DOT did
${ }^{1} \mathrm{~F}$ Sakhinia, ${ }^{2} \mathrm{~J}$ Robinson, ${ }^{2} \mathrm{R}$ McCann, ${ }^{3} \mathrm{C}$ Bell, ${ }^{3} \mathrm{~F}$ Child, ${ }^{1} \mathrm{C} S$ Murray. ${ }^{1}$ University of Manchester, Manchester, UK; ${ }^{2}$ Greater Manchester Health Protection Unit, Manchester, UK; ${ }^{3}$ Central Manchester and Manchester Children's Hospitals Foundation Trust, Manchester, UK

Background Tuberculosis cases in Greater Manchester (GM) have increased annually since 2004. The Paediatric TB clinic at Royal Manchester Children's Hospital has grown since its inception in 2004 , but it is unclear as to whether this is due to a true increase in cases or a change in referral patterns. At the same time the uptake of $B C G$ vaccine is sub-optimal.

Objectives To investigate the incidence, epidemiology and BCG vaccination eligibility and status of childhood tuberculosis cases in GM between 2006 and 2010.

Methods All children ( $\leq 16$ years) notified through the Enhanced Tuberculosis Surveillance System between 1 January 2006 and 31 December 2010 were identified. Vaccination records were obtained from Primary Care Child Health Systems. Missing data were supplemented with examination of case-notes. Eligibility for BCG vaccine was determined by place of birth and ethnicity.

Results 215 children (89 male; mean age 8.8 years) were notified over the 5 years. A rise of $64.5 \%$ in overall number of cases was reported from 2006 to 2010. Pakistanis comprised 39.1\% of TB cases, Black Africans $28.8 \%$ and white British $14.9 \%$. The majority of children were UK-born (60.5\%). Of non-UK born cases $67.1 \%$ entered the UK within 2 years of their diagnosis. Of 130 UK-born children, 111 were deemed eligible for BCG vaccination. Of these 85 (75.6\%) received the vaccine. Of 85 children born outside the UK, vaccination status could not be determined in 8 , and one child was ineligible for vaccination. Vaccination was confirmed in $53 \%$ of nonUK born children (BCG record or BCG scar). In children who had not received BCG, although the number of cases was very small, a CNS involvement) was identified.

Conclusion There has been a significant rise in incidence of Paediatric $\mathrm{TB}$ in GM over the last 5 years. The reason for this remains unclear However, BCG vaccination uptake rates were poor (75\% of UK born individuals and $67 \%$ overall). Systems for identifying eligible children and immunising them need to be reviewed and strengthened both for high risk neonates and children entering the country.

\section{P53 OUR EXPERIENCE OF AVOIDING UNNECESSARY BRONCHOSCOPIES BY USE OF SPUTUM INDUCTION FOR THE INVESTIGATION OF SUSPECTED TUBERCULOSIS}

doi:10.1136/thoraxjnl-2011-201054c.53

T Gorsuch, L Wilson, J L Hoyle. North Manchester General Hospital, Manchester, UK

Introduction The majority of cases of pulmonary tuberculosis (pTB) are diagnosed by microscopy and culture of sputum. When a patient is unable to produce sputum spontaneously, further procedures are required to obtain suitable samples for examination. There is debate threefold higher risk of more severe forms of infection (military, 